\title{
基于非接触式电容传感的人体运动意图识别
}

\author{
王启宁 ${ }^{1,2}$ 郑恩昊 ${ }^{3}$ 许东方 ${ }^{1}$ 麦金耿 $^{1}$ \\ (1. 北京大学工学院 北京 100871 ; \\ 2. 北京大学工程科学与新兴技术高精尖创新中心 北京 100871 ; \\ 3. 中国科学院自动化研究所复杂系统管理与控制国家重点实验室 北京 100190)
}

\begin{abstract}
摘要: 人体运动意图的准确可靠识别是人机共融中的关键问题之一。针对现有研究中的局限和不足, 提出了全新的非接触式 电容传感方法。该方法以金属电极不接触皮肤的方式测量肌肉收缩信号。介绍了电容传感的原理，分析了基于该方法测量肌 肉收缩形状变化的机理。分别介绍了非接触式电容传感方法在小腿智能动力假肢控制和上肢运动识别中的应用。针对下肢智 能假肢控制, 提出了基于非接触式电容传感的运动模态以及模态切换的识别。为了进一步提高系统的可穿戴性, 提出了基于 柔性可延展液态金属电极的电容传感系统并进行了初步的试验验证; 针对上肢运动识别, 首先介绍了用于腕关节离散运动模 式的识别研究, 其次介绍了基于电容传感对连续握力的识别和估计, 证实了电容传感这一全新方法在上肢运动识别中的可行 性。未来会在穿戴式机器人控制以及协作性机器人模仿学习中对电容传感方法进行更深入的研究。
\end{abstract}

关键词: 人体运动意图识别; 非接触式电容传感; 下肢智能假肢; 上肢运动识别

中图分类号: TG156

\section{Noncontact Capacitive Sensing Based Human Motion Intent Recognition}

\author{
WANG Qining $^{1,2}$ ZHENG Enhao $^{3} \quad$ XU Dongfang $^{1} \quad$ MAI Jingeng $^{1}$
}

(1. College of Engineering, Peking University, Beijing 100871;

2. Beijing Innovation Center for Engineering Science and Advanced Technology (BIC-ESAT), Beijing 100871;

3. The State Key Laboratory of Management and Control for Complex Systems, Institute of Automation,

Chinese Academy of Sciences, Beijing 100190)

\begin{abstract}
Accurate and reliable recognition of human motion intent is an important issue in the field of tri-co robotics. A noncontact capacitive sensing approach is proposed which can measure muscle contraction signals by freeing human skin from contacting to metal electrodes. The method is proposed to address the issues in biological-signal-based human motion sensing. We then introduced four case studies of noncontact capacitive sensing. The first study involved locomotion transition recognition on transtibial amputees wearing lower-limb robotic prostheses. The second one is the initial study on new capacitive sensing electrodes with liquid metal electrode, the purpose of which is to build a soft elastic capacitive sensing front-end for more compatible dressing. By comparison the last two studies are applications of the capacitive sensing method on human upper limb motion recognition to prove the feasibility of the new method on upper limb motion sensing. The third one is the forearm discrete motion pattern recognition and the last case is the continuous grasp force estimation with the capacitance signals. The results of the studies suggest the promise of the new sensing method. In future works, the noncontact capacitive sensing approach will be further studied for the control of wearable robots and collaborative robots.
\end{abstract}

Key words: human motion recognition; noncontact capacitive sensing; lower-limb intelligent prosthesis; human upper-limb motion sensing

\section{0 前言}

作为共融机器人 ${ }^{[1]}$ 的典型代表, 穿戴式机器人 ${ }^{[2]}$

\footnotetext{
* 国家自然科学基金资助项目(91648207, 61703400)。20180331 收到初稿, 20181121 收到修改稿
}

与协作型机器人 ${ }^{[3]}$ 技术在医疗康复、助残助老, 以 及工业生产等领域中发挥着越来越重要的作用。在 这些领域中, 人与机器人存在着较强的物理交互和 信息交互。实现对人运动意图及时、准确的识别是 这类机器人控制需要解决的核心问题之一。运动意 图识别的性能不仅决定了机器人系统的性能, 而且 
影响了人在运动/作业环境中的安全性。该方向也得 到了领域中研究者越来越多的关注与研究。实现运 动意图的识别有两个关键的环节, 分别为传感方法 与识别算法。传感方法是运动意图识别的第一个关 键环节, 主要目的是有效提取人的运动信息; 第二 关键环节识别算法的主要目的是将传感系统得到的 信号转换、解码为相应的运动意图信息。传感信号 的类型和特征直接决定了识别算法的设计进而影响 了最终的识别性能, 因此, 传感方法在运动意图识 别中起着至关重要的作用。

基于现有的技术, 按照测量方式, 人体运动信 息的传感手段主要包括非穿戴式传感和穿戴式传感 方法, 非穿戴式传感方法主要在特定的场地环境中 测量人的运动信息, 例如光学传感 ${ }^{[4]}$ 系统和平台式 测力系统 ${ }^{[5]}$ 。穿戴式传感系统以可穿戴在人体表面 的方式测量人的运动信息。相比前者该传感方式不 仅可以突破测量场地限制, 而且可以测量更丰富的 人体运动信息。可通过穿戴式方法测量的人体运动 信号主要包括神经信号 ${ }^{[6]}$ 和机械信号 ${ }^{[7]}$ 。其中, 神经 信号为神经中枢信息的直接反映或者次级反映, 主 要包括脑电信号、肌肉电信号和其他外周神经类电 信号等; 机械信号主要反映人运动学与动力学信息, 包括关节角度、加速度、地面交互力等; 在机械信 号相关研究中, 一部分研究者专门为测量人体运动 研制传感系统, 测量足底压力分布、人机交互力分 布等反映生物力学特性的机械信号 ${ }^{[8]}$ 。

神经信号反映了人神经中枢的活动信息, 具有 延时小和准确度高的优点。该领域很多研究者也利 用了神经信号的特点, 采用神经信号与机械信号融 合的方法来得到更加准确和可靠的人体运动意图识 别 ${ }^{[9]}$ 。按神经信号传感系统测量前端与人体的相对 位置, 可以分为植入式和非植入式两种方法。植 入式方法包括植入式脑电、植入式外周神经信号 等 ${ }^{[10-11]}$, 该方法能够得到较为直接的神经链路信息, 但是由于植入式方法对生物组织存在潜在伤害和安 全性问题, 目前在人体上实际应用较少。相比之下, 非侵入式生物信号传感方法得到了大多使用者的接 受。表面肌肉电信号(简称表面肌电)是目前应用最 广泛的神经信号。表面肌电是肌肉收缩时产生的电 信号在皮肤表面的反映, 能够以非侵入皮肤的方式 测量肌肉收缩中的运动意图信息。由于骨骼肌是人 体运动神经链路中的执行单元, 因此表面肌电包含 了丰富的人体运动信息。现有基于表面肌电的研究 包括了针对智能动力假肢的运动模态识别 ${ }^{[12-14]}$ 、针 对外骨骼和动力矫形器 ${ }^{[15-18]}$ 的人机接口以及针对协 作型机器人的模仿学习 ${ }^{[19-21]}$ 。
然而, 表面肌电在实际应用中存在着一些局限。 首先, 表面肌电属于微弱的生物电信号(信号幅值为 $\mu \mathrm{V}$ 级别), 为获得较高的信号质量, 在测量时金属 电极必须紧贴测量位置处的皮肤表面, 运动中皮肤 表面的汗液会对信号质量造成严重的干扰 ${ }^{[22]}$ 。其 次, 表面肌电信号对测量位置比较敏感, 不同测量 位置会造成信号的波形发生变化。因此, 为保证运 动识别任务的稳定性, 每次使用之前需要重新标定 测量位置 ${ }^{[23]}$ 。传感方法的局限性影响了后续的识别 算法设计, 进而影响了整个识别系统的性能。实现 可靠稳定的意图识别仍然充满了挑战。

针对现有表面肌电信号的局限性, 本团队提出 了人体电容传感的方法, 该方法以金属电极不接触 皮肤的方式测量肌肉收缩信号 ${ }^{[26]}$ 。本文首先介绍非 接触式电容传感的原理, 其次分别介绍了电容传感 在下肢智能动力假肢控制和上肢人机协作任务中的 应用。最后, 分析了电容传感方法的未来发展趋势 和面对的挑战。

\section{1 非接触式电容传感原理}

人体的骨骼肌由肌腹、肌腱和相关的神经血管 等组成, 其中肌腹由肌肉组织构成, 具有收缩力; 肌腱将肌腹连接到骨骼上, 具有弹性但不能产生收 缩力。骨骼肌的肌肉组织包括骨骼肌细胞和间质, 骨骼肌细胞呈细长纤维状机构, 因此又称为肌纤维。 一块骨骼肌即又多束肌纤维并排组成, 每一条肌纤 维又由多条肌原纤维排列而成, 肌原纤维的收缩引 起了最终整条骨骼肌肌腹的收缩。肌腹收缩通过连 接到骨骼的肌腱带动了关节的运动。骨骼肌的收缩 是一个复杂的生理反应, 包括了几个关键的环节:

首先, 骨骼肌细胞接收到神经中枢的刺激产生兴奋 性点冲动; 其次, 电冲动引起了肌肉中离子浓度发 生一系列变化; 第三, 肌纤维中的粗肌丝与细肌丝 结合, 拖动细肌丝向中央方向滑动。最后, 肌节发 生变化, 肌原纤维收缩, 引起骨骼肌收缩。在肌肉 的收缩过程中, 除肌丝的相对滑行引起了最终肌腹 形状的变化, 如维度增粗、长度缩短。在实际的运 动中, 多条骨骼肌协同收缩, 肌肉的形变挤压软组 织, 加上重力和惯性等作用, 最终引起肢体表面的 形状变化 ${ }^{[24]}$ 。肢体表面形状的变化是多个因素共同 作用的结果, 虽然目前很难建立形变和具体运动的 准确映射, 但是基于超声肌肉图像的研究证实了肌 肉形变包含着丰富的人体运动信息 ${ }^{[25]}$ 。

本团队提出了非接触式电容传感方法的原理即 利用一系列等效电容, 记录人运动中的肌肉形变规 
律, 从电容信号中提取运动意图信息。非接触式电 容传感测量前端的基本原理如图 1 所示。在系统中, 金属电极嵌入到穿戴系统前端的内侧(靠近人体一 侧), 与人体皮肤之间有一层绝缘层。人体、金属电 极、绝缘层构成了一个等效平板电容。两个电极分 别为人体和金属电极。人在运动中, 肌肉收缩产生 的形变会造成肢体表面的形变, 造成人体与金属电 极之间的相对位置发生变化, 造成等效平板电容的 电极距离发生变化, 进而引起电容值的变化。穿戴 系统前端为金属电极的载体, 根据具体的应用场景 确定, 例如针对下肢智能假肢, 穿戴系统前端为假 肢的接受腔。在实际的穿戴中, 绝缘层一般为被试 的衣物和穿戴前端的保护层。

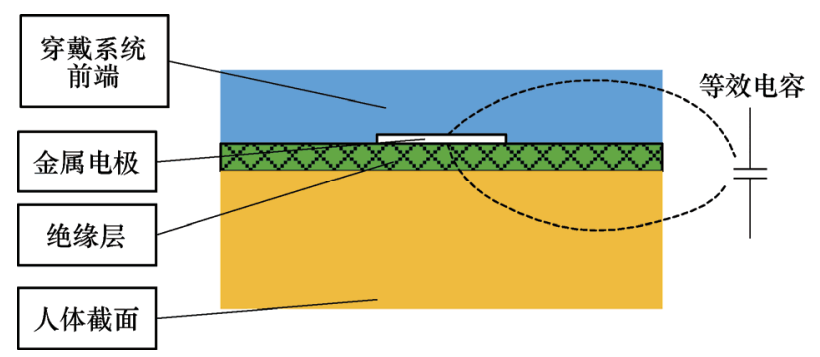

图 1 非接触式电容传感基本原理示意图

非接触式电容传感系统通过测量其充放电时间 来记录电容信号, 我们将放电电阻 $R$ 与耦合电容 $C_{\text {body }}$ 构成串联回路, 并在回路中施加方波信号, 在 方波信号的上升沿, 电容迅速充电至最高电压 $V_{\mathrm{s}}$, 在方波下降沿, 电容开始通过放电电阻 $R$ 放电, 根 据电容放电公式, 当最终电容电压 $V_{\mathrm{t}}=0.37 \times V_{\mathrm{s}}$ 时, 对应的放电时间 $t=R \times C_{\mathrm{body}}$ 。而该时间与电容的容值 呈正比, 通过记录该放电时间便可以得到相应的电 容值。为了精确测量电容信号的变化, 本课题采用 了 $\mathrm{CTD}$ (电容-时间转换) 模块记录电容充放电时间 的变化。CTD 模块内包括选通单元、施密特出发单 元和运算单元。选通单元的功能是选通待测电容 $C_{\mathrm{i}}$ 与放电电阻 $R$ 构成放电回路, 施密特触发单元的作 用是把电容充放电波形转换为方波, 运算单元的作 用是通过计数器来记录电容重放点时间获得电容 值。在电容信号测量电路中包含了一个参考电容 $C_{\mathrm{r}}$, 参考电容和放电电阻的值根据待测电容的大小设 定, 防止计数器溢出。计数器根据计数频率测量待 测电容 $C_{\mathrm{i}}$ 与参考电容 $C_{\mathrm{r}}$ 之间的充放电时间差值, 运算单元根据该时间差值来计算出待测电容与参考 电容的比值, 进而计算出实际的待测电容值。电容 信号系统最终输出频率为 $100 \mathrm{~Hz}$, 有效分辨率为 $21 \mathrm{bit}$, 电容信号的量程为 $0.125 C_{\mathrm{r}} \sim 8 C_{\mathrm{r}}$ 。根据下肢 运动与上肢运动识别中电容传感测量前端调节参考
电容 $C_{\mathrm{r}}$, 实现对信号的测量。在试验中, 试验数据 主要包括电容信号与机械信号(足底压力信号和惯 性信号), 机械信号可为电容信号进行数据标定作为 参考与辅助。在系统中, 我们设计了信号同步电路 将所有的数据以 $100 \mathrm{~Hz}$ 的采样频率发送至电脑并 存储作为后续分析的试验数据。

\section{2 电容传感在智能下肢假肢中的应用}

\section{1 下肢运动模态识别研究}

本团队针对智能动力小腿假肢的控制, 提出了 非接触式电容传感的方法 ${ }^{[26-27]}$ 。智能下肢假肢控制 一般采用分层控制策略, 高层识别运动意图、中层 将运动意图转换为控制策略、底层实现对驱动系统 的控制。因此下肢运动意图的识别对实现残疾人穿 戴智能假肢流畅运动至关重要。人的运动模态指不 同的运动状态，例如站立、平地行走、上下楼梯、 跨越障碍物等。人在不同的运动模态下，下肢肌肉 的参与情况不同, 虽然在大多数情况下参与的肌肉 群基本一致, 但是每种肌肉起作用的步态阶段, 以 及收缩强度均不同 ${ }^{[28]}$ 。因此需要设计有效的传感系 统, 使之能够尽可能多获取下肢运动信息、且对残 疾人带来尽可能小的运动干预。

残疾人接受腔是假肢的重要组成部分, 它一方 面直接与残疾人的残端接触, 另一方面连接着假肢 的驱动系统。残疾人身体重要通过接受腔承担, 同 时通过接受腔带动着假肢的运动。残疾人在截肢后, 残肢成为了身体与地面直接交互的部位。在落地期, 残肢与接受腔之间的交互力取决于身体的重量和重 心移动; 在摆动期, 残肢与接受腔之间的交互力比 落地期微弱得多, 它主要取决于残端的形变以及摆 动过程中的加速度。非接触式电容传感系统的测量 原理如图 2a 所示, 电极片放置在假肢的接受腔内, 位于内祄套和接受腔之间，其中人体为导体，人体 与电极片之间构成了耦合电容的两个电极, 中间的 内祄套(材料一般包括硅胶、橡胶、尼龙等)为电容 的电介质。在下肢运动过程中, 残肢与接受腔的交 互力, 以及残肢的肌肉形变都会造成内祄套厚度的 改变(等效电容两个电极之间距离改变), 进而改变 电容信号。通过记录该电容信号就可以得到相应的 运动信息。我们在接受腔内一共设置了 6 个电容信 号通道, 其位置分别为: (1) 胫骨前端, 髌韧带下侧; (2) 残肢末端前侧, 胫骨末梢; (3) 残肢末端后侧, 腓肠肌下侧对应位置; (4) 腓肠肌上侧; (5) 股骨内 测; (6) 股骨外侧。为了精确测量电容信号的变化, 我们设计了电容-时间转换模块记录电容充放电时 
间的变化。电容信号的数字采样频率为 $100 \mathrm{~Hz}$ 。由 于电容信号值微弱, 且存在着杂散电容等干扰, 原 始信号存在着高频噪音和低频漂移。我们设计了三 级滤波器来去除噪音, 分别为中位值滤波、一阶直 流陷波器和 2 阶巴特沃斯低通滤波器。

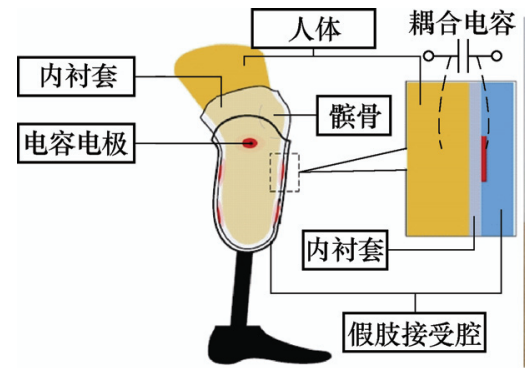

(a) 原理示意图

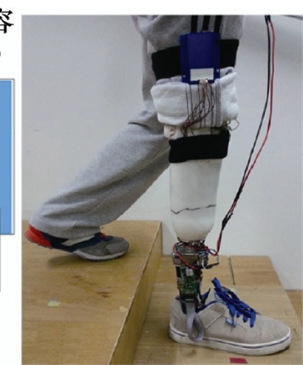

(b) 实际穿戴图
图 2 用于智能小腿假肢的非接触式电容传感示意图 ${ }^{[26-27]}$

非接触式电容传感系统在集成到智能小腿假 肢上后, 我们对其在连续运动模态识别中的性能做 了验证。在试验中, 6 名单侧小腿截肢的残疾人被 招募。在试验中，所有的被试穿戴自己的假肢接 受腔, 通过连接杆连接至智能小腿假肢 PKURoboTPro $^{[29]}$ (穿戴效果如图 2b 所示)。非接触式电 容系统的测试前端放置在接受腔和内祄套之间, 电 路的信号地通过一个电极片放置到内衬套内部, 接 触残疾人皮肤将人体与电路共地。所有残疾人被试 在参加试验之前均提供了知情同意书。试验测试了 在日常生活中常见的 6 种运动模态下连续行走, 一 共测量了 6 种运动模态和 10 种运动模态之间的切 换。6 种运动模态包括: 静止站立 $(\mathrm{St})$ 、平地行走 $(\mathrm{W})$ 、上楼梯 $(\mathrm{SA})$ 、下楼梯 $(\mathrm{SD})$ 、上斜坡 $(\mathrm{RA})$ 和下 斜坡(RD)。运动模态之间的切换包括: 静止站立和 平地行走之间的切换 $(\mathrm{St} \leftrightarrow \mathrm{W})$ 、平地行走和上下楼 梯之间的切换 $(\mathrm{SA} / \mathrm{SD} \leftrightarrow \mathrm{W})$ 以及平地行走和上鞋斜 坡之间的切换 $(\mathrm{RA} / \mathrm{RD} \leftrightarrow \mathrm{W})$ 。试验中, 我们采集了 不同 “切换脚” 的数据, “切换脚” 即发生运动模 态切换的一侧脚, 比如从平地走切换到上楼梯, 被 试既可以左脚先迈上楼梯, 也可以右脚先迈上楼 梯, 因此发生切换的这一侧被称为 “切换脚”。每 个被试的试验包括 15 组测量, 每一组测量中, 被 试在试验平台上一共行走四次, 分别包括两个方向 和两个 “切换脚” 的测量。

传感系统采集的信号主要包括 6 个通道的电容 信号和 10 个通道的两个惯导信号。所有信号的采样 频率为 $100 \mathrm{~Hz}$ 。我们使用滑动窗的方法来分割数据, 滑动窗长度为 $250 \mathrm{~ms}$, 滑动窗滑动的步长设置为 10 $\mathrm{ms}$, 即一个采样周期。利用滑动窗进行连续运动意 图识别的优点是能够在每一个采样时刻输出运动模
态的决策, 当运动模态发生切换时, 滑动窗能够迅 速地抓住运动变化的信息, 在关键时刻之前识别出 即将切换的运动。在每个滑动窗上，我们分别针对 电容信号提取了 6 个时域特征值, 针对假肢机械信 号提取了 4 个时域特征值。本研究使用了级联分类 器来进行运动模态切换的识别, 第一级分类器的功 能是识别出 “静止站立” 和 “运动”, 第二级分类器 的功能是将前一级分类器的输出(当分类器果为 “运 动”)再细分为各个运动模态。第一级的分类算法为 二次判别分析 $(\mathrm{QDA})$, 第二级分类算法为支持向量 机(SVM)。

第一级识别任务相对简单，仅区分静止站立和 行走，依靠 QDA 分类算法，所有被试的离线识别 准确率为 $100 \%$, 所有的运动起始和运动终止均可 以准确识别。第二级分类算法的目的是将运动的数 据细分为各个运动模态。识别结果的混淆矩阵(混淆 矩阵的详细定义见文献[26])如图 3a 所示。我们分别 在行走的摆动期和落地期单独训练了分类器, 所有 运动模态平均识别准确率为 $95.8 \%$, 摆动期和落地 期的识别率基本一致，并且在每种步态的识别结果 中也表现出一致的规律。在摆动期，上下楼梯 $(\mathrm{SA} / \mathrm{SD})$ 比上下斜坡 $(\mathrm{RA} / \mathrm{RD})$ 的识别准确率要高, 但

\begin{tabular}{|c|c|c|c|c|c|c|}
\hline \multicolumn{2}{|c|}{} & \multicolumn{5}{|c|}{ 识别出的运动模态 } \\
\hline 步态阶段 & 实际运动模态 & W & SA & SD & RA & RD \\
\hline \multirow{3}{*}{ 摆动期 } & W & 95.9 & 0.2 & 0.1 & 2.3 & 1.5 \\
& $\mathrm{SA}$ & 1.1 & 97.9 & 0.1 & 0.8 & 0.1 \\
& $\mathrm{SD}$ & 1.4 & 0.3 & 97.1 & 0.2 & 1.1 \\
& $\mathrm{RA}$ & 5.7 & 0.6 & 0.1 & 93.6 & 0.1 \\
& $\mathrm{RD}$ & 4.9 & 0.0 & 0.5 & 0.1 & 94.5 \\
\hline \multirow{3}{*}{ 落地期 } & $\mathrm{W}$ & 93.2 & 4.2 & 2.0 & 0.2 & 0.4 \\
& $\mathrm{SA}$ & 4.2 & 94.4 & 0.7 & 0.4 & 0.3 \\
& $\mathrm{SD}$ & 4.2 & 1.0 & 94.1 & 0.1 & 0.6 \\
& $\mathrm{RA}$ & 0.8 & 0.4 & 0.1 & 98.7 & 0.0 \\
& $\mathrm{RD}$ & 1.3 & 0.1 & 0.1 & 0.0 & 98.5 \\
\hline
\end{tabular}

(a) 识别准确率混淆矩阵

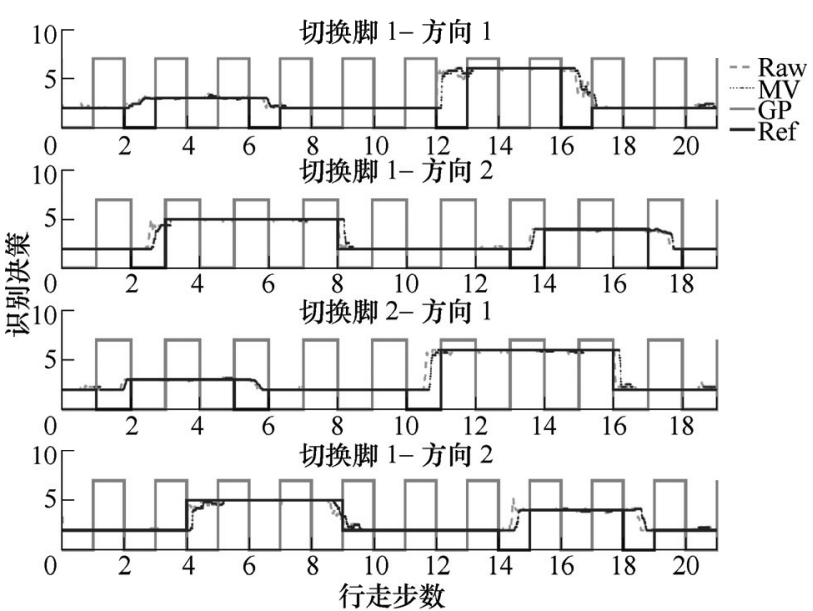

(b) 实施结果示意图 ( 数据来源于被试 3)

图 3 运动模态切换识别结果 ${ }^{[27]}$ 
是在落地期正好相反。该研究也评估了伪实时识别 结果, 如图 3 下侧所示。图中横轴表示按照时间归 一化的步态周期数目, 纵轴表示识别决策, 其中 2 表示平地行走、 3 表示上楼梯、 4 表示下楼梯、 5 表 示上斜坡、6 表示下斜坡。灰色实线表示步态阶段 (GP)，0 表示摆动期、7 表示落地期。虚线段表示原 始的识别决策(Raw), 描点线表示经过后处理的识别 决策(MV), 黑色实线表示期望的识别的识别决策 (Ref)。识别决策按照每个步态周期百分比进行归一 化, 同时按照所有的试次进行了平均。可以看出, 大多数识别决策均可以在步态发生切换的落地时刻 之前识别出。

\section{2 基于液态金属电极的电容传感系统}

在第 2.1 节中所述的电容传感系统, 电极采用 了柔性印制电路板, 此外, 为提高测量前端的延 展性, 本团队研究了基于柔性材料的电容传感系 统。该柔性材料为液态金属, 液态金属作为一种 导体, 由于其良好的液态可变形、柔性等性能, 被广泛应用在电子机械等领域 ${ }^{[30-31]}$ 。液态金属通 常为镓铟 $(G a I n)$ 等合金, 具有高导电性、低熔化温 度 $\left(<15{ }^{\circ} \mathrm{C}\right)$ 的特点 ${ }^{[32]}$ 。PDMS (聚二甲基硅氧烷)是 一种常见的柔性祄底材料, 具有良好的力学特性。 我们设计制作了基于 PDMS 祄底的液态金属电极, 详见图 4 右侧。

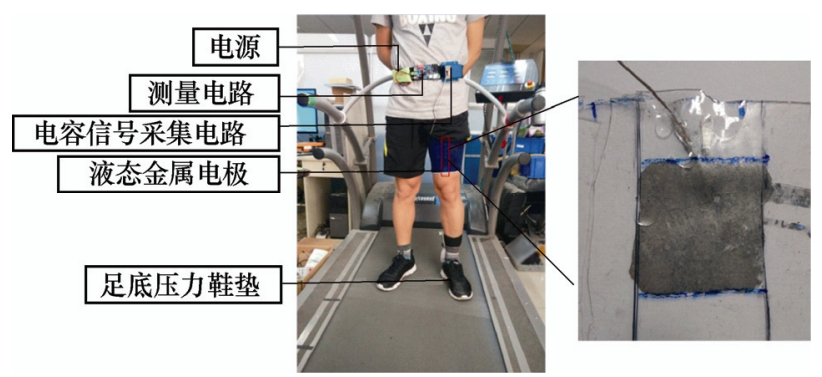

图 4 液态金属电容传感电极与穿戴示意图。

基于液态金属电极我们开展了针对多种地形的 运动模式识别试验。图 4 左侧是被试穿戴液态金属 电极开展试验的示意图。六个液态金属电极分别置 于大腿前侧和后侧, 每侧三个电极呈上中下排列。 柔性布质护膝被套在大腿上, 使电极和皮肤紧密贴 合。同时, 被试需要在同侧穿戴足底压力鞋垫, 该 压力鞋垫可以测量被试行走过程中的足底压力信 号, 进而进行步态划分。电容信号采集方法与第 2.1 节中相同。一位健康被试参与了该运动模式识别试 验。被试在跑步机上分别完成平地行走(Level ground walking, LG), 上斜坡(Ramp ascending, RA), 下斜坡(Ramp descending, RD) 三种地形试验, 每类 地形下被试以 $1.0 \mathrm{~m} / \mathrm{s}$ 的速度持续行走 $3 \sim 4 \mathrm{~min}$ 。
我们首先分析了该液态金属电极电容信号的周 期性(重复性)。我们对平地行走的数据进行了步态 划分, 然后将电容数据进行周期上的归一化。图 $5 \mathrm{a}$ 显示了平地行走数据归一化到一个周期后的规律变 化。六个子图代表运动过程中六个液态金属电极信 号的变化, 图中实线代表不同周期同一步态时刻的 电容信号平均值, 阴影部分代表相应的标准差。从 图 3 可以看出液态金属电极电容信号呈现出良好的 周期性。图 $5 \mathrm{~b}$ 的两个混淆矩阵分别是利用液态金属 电极和铜电极采集电容信号进行三种运动模式识别 的结果。从结果中可以看出, 液态金属电极可以取 得同铜电极相当的结果。采用液态金属电极, 三种 地形分别获得了 $99.0 \% 、 99.2 \% 、 99.99 \%$ 的识别准确 率。数据的周期性规律变化和识别结果表明, 采用 基于液态金属电极的电容传感进行运动模式识别是 可行的。相对于普通金属电极，液态金属电极可以 承受更大的拉伸挤压等变形, 其良好的柔性使得在 更复杂环境下的穿戴成为了可能。
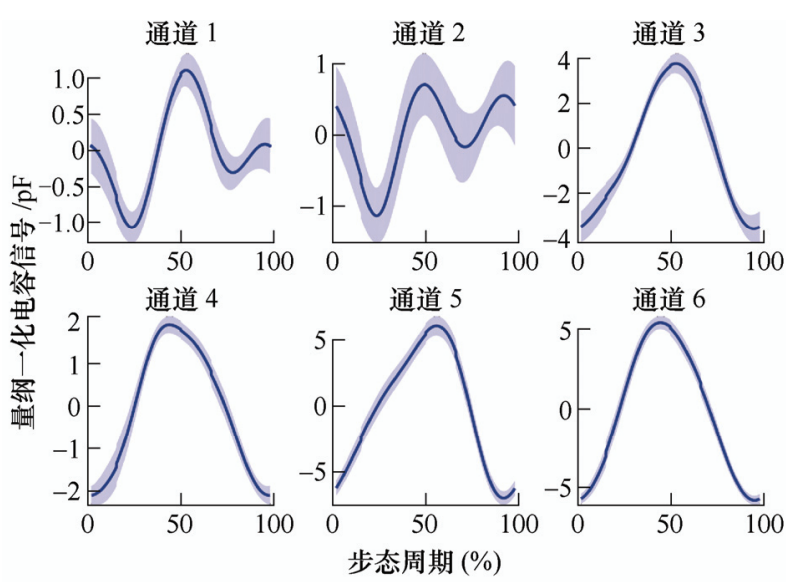

(a) 步态归一化后的电容信号

\begin{tabular}{|l|l|l|l|}
\hline \multicolumn{5}{|c|}{ 液态金属电极 } \\
\hline & 平地行走 & 上斜坡 $\left(10^{\circ}\right)$ & 下斜坡 $\left(10^{\circ}\right)$ \\
\hline 平地行走 & $99.00 \%$ & $0.34 \%$ & $0.66 \%$ \\
\hline 上斜坡 $\left(10^{\circ}\right)$ & $0.00 \%$ & $99.20 \%$ & $0.80 \%$ \\
\hline 下斜坡 $\left(10^{\circ}\right)$ & $0.00 \%$ & $0.01 \%$ & $99.99 \%$ \\
\hline \multicolumn{5}{|c|}{ 普通铜电极 } \\
\hline & 平地行走 & 上斜坡 $\left(10^{\circ}\right)$ & 下斜坡 $\left(10^{\circ}\right)$ \\
\hline \multicolumn{1}{|c}{ 平地行走 } & $95.99 \%$ & $3.98 \%$ & $0.04 \%$ \\
\hline 上斜坡 $\left(10^{\circ}\right)$ & $1.28 \%$ & $98.72 \%$ & $0.00 \%$ \\
\hline 下斜坡 $\left(10^{\circ}\right)$ & $0.00 \%$ & $0.43 \%$ & $99.57 \%$ \\
\hline
\end{tabular}

(b) 识别准确率的混淆矩阵

图 5 基于液态金属电极的试验结果

\section{3 电容传感在上肢运动识别中的应用}

\section{1 上肢运动模式识别研究}

人类日常行为中的下肢运动与上肢运动有显著 不同的特点, 下肢以周期性运动为主, 肌肉的收缩 
模式体现出了很强的周期性, 每个步态周期的不同 阶段也体现出明显的生物力学特性和肌肉收缩模 式; 不同的运动模式下的下肢运动也体现出了比较 明显的生物力学特性差异; 因此, 下肢运动意图识 别任务的重点除准确性外, 还强调实时性。相比下 肢运动, 上肢运动任务中没有较多的周期性运动, 日常生活动上肢运动类型更加多样化, 也更加复杂, 相应的肌肉收缩模式也更加复杂和多样化; 相比下 肢运动的神经控制方式, 上肢运动更多为直接的意 图控制, 肌肉收缩模式大多为任务或目标驱动 (target based), 识别任务也更强调识别的准确性和任 务复杂性。电容信号反映出肌肉收缩特征以及识别 任务都存在明显的差异。

用于下肢假肢的电容传感测量前端是将金属电 极嵌入假肢的接受腔中, 金属电极、皮肤和内祄套 构成耦合电容, 肌肉形变以及残端与接受腔交互力 都会引起电容信号变化。与下肢运动不同, 上肢运 动没有相对周期性的与外界的物理交互(交互力)。 因此, 电容传感测得的信息主要为肢体形变信息。 我们拟设计的用于上肢运动测量的电容传感测量系 统前端结构如图 6 所示。电容测量环位于前臂靠近 肘关节位置, 距离肘关节大概 $1 / 3$ 前臂长度。电容 测量环的结构如图 4 左侧所示, 其主体是热塑材料 制成的热塑环, 热塑环根据穿戴者的肢体形状塑形, 热塑环内侧集成了多个金属电极片, 在金属电极片 上覆盖一层硅胶薄膜作为金属电极与外界的绝缘 层。电容传感方法的测量原理如图 6 右上侧所示, 一个金属电极、硅胶绝缘层、人体构成了一个等效 电容, 运动过程中肌肉收缩引起的肢体形变对硅胶 薄膜的挤压, 引起等效电容两电极之间距离的变化, 进而引起电容值的变化。每个电容测量环分别设置 一缺口, 缺口位于肢体内侧(靠近人体中线一侧), 缺口用可调节松紧度的绑带固定。电容信号采集系

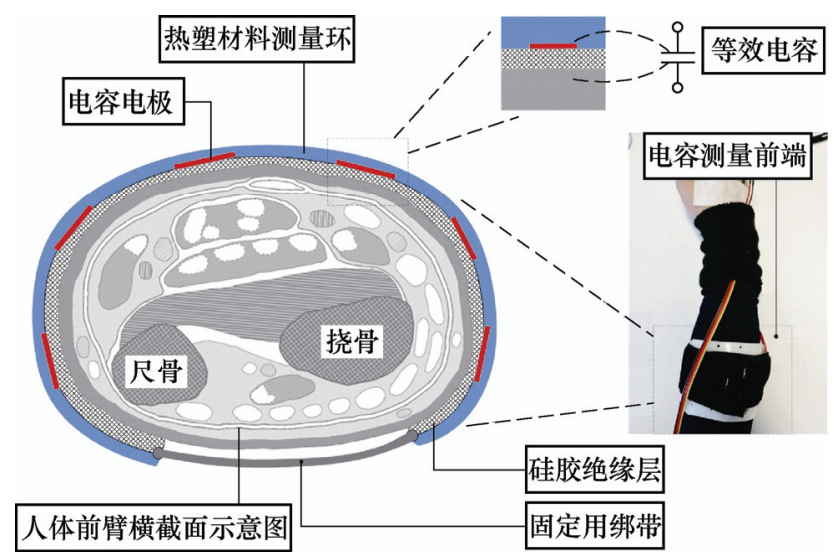

图 6 用于人前臂的电容传感前端示意图
统与第 2.1 节中相似, 采用了测量电容充放电周期 来得到实际的电容值, 采样频率为 $100 \mathrm{~Hz}$ 。我们设 计了一上位机界面用于采集和存储数据。

为初步验证基于电容传感的上肢运动模式识 别, 本研究招募了 3 名健康男性被试。所有的被试 填写了知情同意书。被试的平均年龄为 24.3 岁, 平 均右前臂长度为 $22.7 \mathrm{~cm}$, 右前臂平均最大周长为 $24.3 \mathrm{~cm}$ (取前臂最粗处测量)。在试验中共测量了 9 种运动模式, 分别是腕关节屈 $(\mathrm{WF}) 、$ 伸 (WR)、旋前 $(\mathrm{WP}) 、$ 旋后 $(\mathrm{WS}) 、 尺$ 偏 $(\mathrm{UD}) 、$ 桡偏 $(\mathrm{RD}) 、$ 握拳 $(\mathrm{F}) 、$ 伸掌 $(\mathrm{P})$ 和放松 $(\mathrm{R})$ 。在试验之前, 我们根据每名被试 被测前臂的形状制作了电容测量环。在试验中，每 种运动模式测量了 5 个试次。在每个试次中, 被试 开始于放松状态, 手掌放松位于中立位(手掌与地面 基本垂直), 几秒钟后, 根据提示运动至相应的运动 模式并保持 $10 \sim 12 \mathrm{~s}$ ，然后根据提示回到放松状态 保持 $3 \sim 4 \mathrm{~s}$ 后该试次结束。我们采用了滑动窗的方 法分割数据, 滑动窗长度为 $200 \mathrm{~ms}$, 在每个滑动床 上我们计算四个时域特征值, 分别为平均值、标准 差、最大值和平均斜率。分类算法为 QDA 算法, 评价方法为 5 份留一交叉检验, 每个试次轮流作为 测试组。识别的结果呈现为混淆矩阵, 混淆矩阵中 每个元素代表了识别准确率/误差率。三名被试的平 均识别结果如图 7 所示。三名被试分别表示 $\mathrm{S}_{1} 、 \mathrm{~S}_{2}$ 和 $S_{3}$, 平均识别率的混淆矩阵表示为 AVE。三名被 试的平均识别准确率(混淆矩阵对角线求平均)分别 为： $85.8 \% 、 85.9 \%$ 和 $91.8 \%$ 。通过比较每个被试的 每个运动模式, 我们发现桡偏、尺偏和伸掌识别准 确率相比其他运动模式要低, 绝大多数识别误差发 生在相应的运动模式和放松状态之间。我们进而统 计了去掉放松状态 $(\mathrm{R})$ 后的识别准确率, 平均识别准 确率为 $99.9 \%$ 。如果在识别中仅统计 WF、WR、 $W P 、 W S$ 和 $R$ 五种运动模式, 识别准确率为 $98.4 \%$ 。 因此, 在前臂运动模式识别中, 不同的运动模式对 识别效果有较明显的影响。

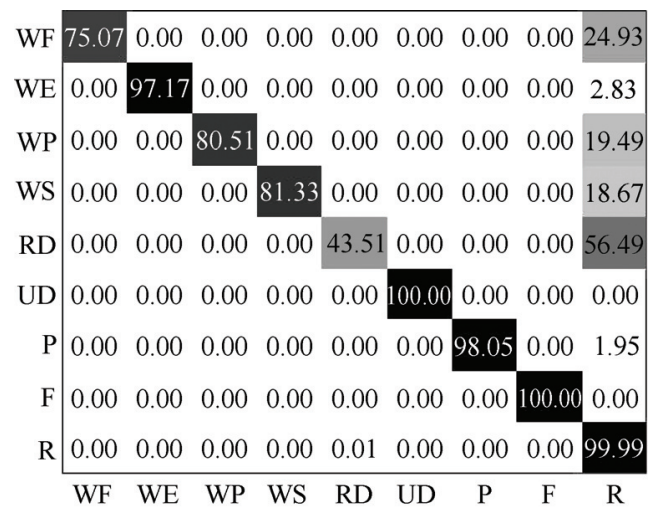

(a) $\mathrm{S}_{1}$ 


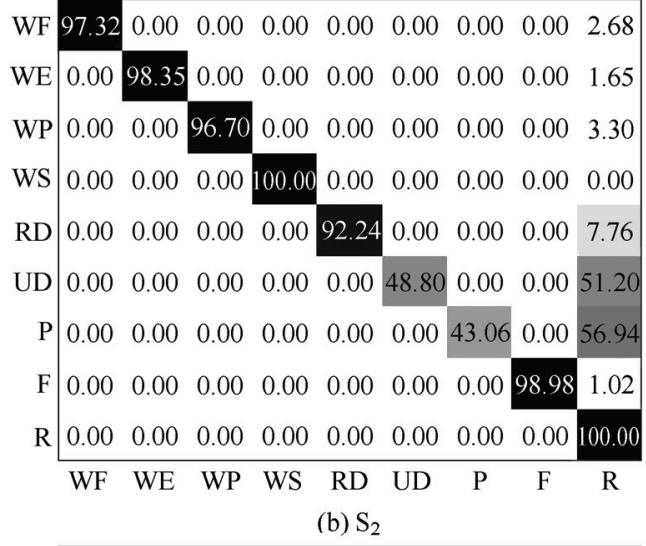

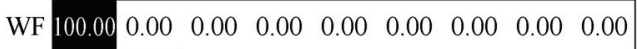

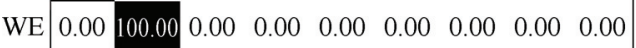
WP $\begin{array}{llllllllll}0.00 & 0.00 & 90.92 & 0.00 & 0.00 & 0.00 & 0.00 & 0.00 & 9.08\end{array}$ WS $\begin{array}{llllllllll}0.00 & 0.00 & 0.00 & 100.00 & 0.00 & 0.00 & 0.00 & 0.00 & 0.00\end{array}$ $\begin{array}{llllllllll}\mathrm{RD} & 0.00 & 0.00 & 0.00 & 0.00 & 50.37 & 0.00 & 0.00 & 0.00 & 49.63\end{array}$ UD $\begin{array}{lllllllll}0.00 & 0.00 & 0.00 & 0.00 & 0.00 & 96.42 & 0.00 & 0.00 & 3.58\end{array}$ $\begin{array}{lllllllllll}\mathrm{P} & 0.00 & 0.00 & 0.00 & 0.00 & 0.00 & 0.00 & 93.41 & 0.00 & 6.59\end{array}$ F $\begin{array}{lllllllll}0.00 & 0.00 & 0.00 & 0.00 & 0.00 & 0.00 & 0.00 & 100.00 & 0.00\end{array}$ $\mathrm{R} \quad \begin{array}{lllllllll}0.00 & 0.00 & 0.00 & 0.00 & 2.16 & 0.00 & 0.00 & 0.01 & 97.82\end{array}$ (c) $\mathrm{S}_{3}$

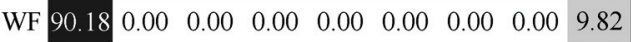
WE $\begin{array}{llllllllll}0.00 & 98.40 & 0.00 & 0.00 & 0.00 & 0.00 & 0.00 & 0.00 & 1.60\end{array}$ WP $\begin{array}{llllllllll}0.00 & 0.00 & 89.20 & 0.00 & 0.00 & 0.00 & 0.00 & 0.00 & 10.80\end{array}$ $\begin{array}{llllllllll}\text { WS } & 0.00 & 0.00 & 0.00 & 93.81 & 0.00 & 0.00 & 0.00 & 0.00 & 6.19\end{array}$ RD $\quad \begin{array}{lllllllll}0.00 & 0.00 & 0.01 & 0.00 & 61.11 & 0.00 & 0.00 & 0.00 & 38.88\end{array}$ UD $\begin{array}{lllllllll}0.00 & 0.00 & 0.00 & 0.00 & 0.00 & 81.60 & 0.00 & 0.00 & 18.40\end{array}$ $\begin{array}{lllllllllll}\mathrm{P} & 0.00 & 0.00 & 0.00 & 0.00 & 0.00 & 0.00 & 77.58 & 0.00 & 22.42\end{array}$ F $\quad \begin{array}{lllllllll}0.00 & 0.00 & 0.00 & 0.00 & 0.00 & 0.00 & 0.00 & 99.60 & 0.40\end{array}$ \begin{tabular}{c|ccccccccc}
$\mathrm{R}$ & 0.00 & 0.00 & 0.00 & 0.00 & 0.66 & 0.00 & 0.03 & 0.02 & 99.29 \\
WF & WE & WP & WS & RD & UD & P & F & R
\end{tabular} (d) 平均

图 7 每个被试的混淆矩阵和平均混淆矩阵

\section{2 握力连续识别研究}

下肢运动模态以步态周期为基本单元，下肢关 节在每个步态周期中的不同步态阶段存在比较明显 的生物力学特性。而上肢运动更多为直接的意图控 制, 除上肢离散运动状态识别(第 3.1 节中介绍), 另 一个重要任务是实现连续运动的识别与估计。非接 触式电容传感测量肌肉收缩信息, 肌肉收缩信号中 除包含关节运动学信息外, 还包含了丰富的力学信 息。例如, 人在不确定的外部干扰环境中通过改变 肌肉的收缩强度来调节关节阻抗, 以克服外界不确 定性的干扰 ${ }^{[33]}$ 。本节介绍的动作为基于非接触式电 容传感的连续握力估计。

在研究中, 3 名男性被试参加了试验, 并填写 了知情同意书。被试平均年龄是 24.7 岁, 平均右前
臂长度为 $23.0 \mathrm{~cm}$, 右前臂平均最大周长为 $25.0 \mathrm{~cm}$ 。 如图 8 所示, 试验测量系统包括电容测量环(试验前 根据每个被试右前臂形状塑形)、信号采集电路和握 力传感器。在该试验系统中, 握力信号与电容信号 同步测量, 数字采样频率为 $100 \mathrm{~Hz}$ 。握力传感器包 括电桥式应变电阻, 测量握力的范围为 $0 \sim 800 \mathrm{~N}$, 我们设计的信号采集电路激励电压为 $10 \mathrm{~V}$, 输出握 力信号的分辨率为 $2.068 \mathrm{mV} / \mathrm{V}$, 通过线性放大至 $0 \sim 3.3 \mathrm{~V}$ 范围后通过模拟数字转换控制器( $\mathrm{ADC}$ )转 换至数字信号, 以与电容信号同步采集和输出。在 试验中, 被试穿戴好电容传感测量前端和握力传感 器, 以一定的速度慢慢增加握力至最大值(每个被试 自己的最大肌肉收缩 MVC)然后回复至初始状态, 休息几秒钟后重复 10 个试次。在每个收缩-放松周 期中, 收缩的时间为 2 5 $\mathrm{s}$ 。在每个被试试验开始 之前, 被试会有一段时间熟悉试验流程, 我们发现 被试相对比较容易控制收缩阶段的速率, 但是很难 平稳控制放松阶段, 因此在后续的分析中, 我们仅 截取了每个试次中握力增加的阶段。在每段数据中, 电容信号的起始点根据握力信号进行了归一化。电 容信号经过了 4 阶巴特沃斯低通滤波器, 握力信号 经过了一阶滞后滤波。我们分别对每个信号通道(共 6 个通道电容信号)分别与同步测量的握力信号进行 二次回归。通过回归决定系数 $R^{2}$ 来评估结果。

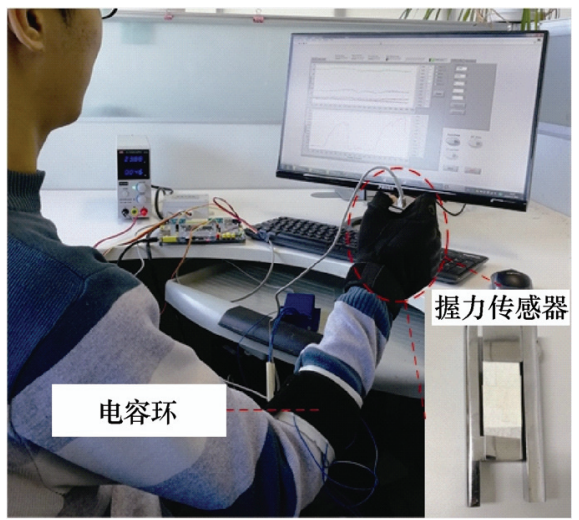

图 8 用于握力识别的试验设置示意图

回归结果如表 1 所示, 从表中可以看出, 不同 被试间存在着很强的个体差异。 $\mathrm{C}_{1}, \mathrm{C}_{2}, \cdots, \mathrm{C}_{6}$ 分别 表示电容传感系统的 6 个通道。对被试 $1, \mathrm{C}_{3}$ 获得 了最好的结果, $R^{2}=0.89$; 被试 2 的通道 2 决定系数

表 1 各个被试和信号通道的回归决定系数

\begin{tabular}{ccccccc}
\hline \multirow{2}{*}{ 被试编号 } & \multicolumn{7}{c}{ 信号通道编号 } \\
\cline { 2 - 7 } & $\mathrm{C}_{1}$ & $\mathrm{C}_{2}$ & $\mathrm{C}_{3}$ & $\mathrm{C}_{4}$ & $\mathrm{C}_{5}$ & $\mathrm{C}_{6}$ \\
\hline 被试 1 & 0.01 & 0.16 & 0.89 & 0 & 0.68 & 0.79 \\
被试 2 & 0.01 & 0.90 & 0.84 & 0.88 & 0.85 & 0.05 \\
被试 3 & 0 & 0.59 & 0.91 & 0.24 & 0.59 & 0.15 \\
\hline
\end{tabular}


为 0.90 , 被试 3 的最好结果发生在通道 3, 决定系 数为 0.91 。回归结果表明, 前臂测量得到的电容信 号与连续握力存在二次相关性。

\section{4 结论}

本文提出了非接触式电容传感的方法, 并将其 应用于下肢运动模态识别和上肢的运动识别。本文 从现有研究的局限出发, 分析了非接触式电容传感 方法的测量原理。同时分别针对下肢运动识别和上 肢运动识别介绍了四种典型的应用。分别为针对下 肢智能假肢控制的运动模态切换识别、针对下肢连 续行走识别的液态金属电极研究、针对上肢运动模 式的识别以及基于电容传感的连续握力估计。本研 究通过试验结果证实了非接触式电容传感在人体运 动意图识别中的有效性和应用于人机共融的可行 性。未来该方向的研究主要集中在如下几个方面。

(1) 基于新型材料的电容传感测量前端研究, 除液态金属电极, 本团队在未来工作中还会研制其 他类型的柔性可延展电容电极, 以达到更好的穿戴 稳定性和可靠性。

(2) 针对穿戴式机器人(下肢智能动力假肢、下 肢外骨骼)和协作型机器人, 开展基于电容传感的人 机接口研究, 提高机器人在人机交互中的性能。

(3) 针对智能动力假肢控制, 通过与外科医学、 神经科学等领域学者进行跨学科交叉研究, 探索全 新术式以增强电容传感的信号特征表达，通过改变 人神经链路提高人机接口的性能。

\section{参 考 文 献}

[1] DING H, YANG X, ZHENG N, et al. Tri-co robot: A Chinese robotic research initiative for enhanced robot interaction capabilities[J]. National Science Review, 2017, 0: 1-3.

[2] PONS J L. Wearable robots : Biomechatronic exoskeletons[M]. John Wiley \& Sons, 2008.

[3] TANG G. The development of a human-robot interface for industrial collaborative system[D]. Cranfield University, 2016.

[4] MAGNENAT-THALMANN N, YUAN J, THALMANN $\mathrm{D}$, et al. Context aware human-robot and human-agent interaction[M]. Springer, 2016.

[5] GIACOMOZZI C. Appropriateness of plantar pressure measurement devices : A comparative technical assessment[J]. Gait \& Posture, 2010, 32(1): 141-144.

[6] HATSOPOULOS N G, DONOGHUE J P. The science of neural interface systems[J]. Annual Review of
Neuroscience, 2009, 32: 249-266.

[7] ROETENBERG D, LUINGE H, SLYCKE P. Xsens MVN: Full 6DOF human motion tracking using miniature inertial sensors[J]. Xsens Motion Technologies BV, Tech. Rep, 2009(1): 1-9.

[8] FONG D T P, CHAN Y Y, HONG Y, et al. Estimating the complete ground reaction forces with pressure insoles in walking[J]. Journal of Biomechanics, 2008, 41(11): 2597-2601.

[9] NOVAK D, RIENER R. A survey of sensor fusion methods in wearable robotics $[\mathrm{J}]$. Robotics and Autonomous Systems, 2015, 73: 155-170.

[10] ENGEL A K, MOLL C K E, FRIED I, et al. Invasive recordings from the human brain: Clinical insights and beyond[J]. Nature Reviews Neuroscience, 2005, 6(1): 35.

[11] DONOGHUE J P. Bridging the brain to the world: A perspective on neural interface systems[J]. Neuron, 2008, 60(3): 511-521.

[12] FARINA D, JIANG N, REHBAUM H, et al. The extraction of neural information from the surface EMG for the control of upper-limb prostheses: Emerging avenues and challenges[J]. IEEE Transactions on Neural Systems and Rehabilitation Engineering, 2014, 22(4): 797-809.

[13] HARGROVE L J, SIMON A M, YOUNG A J, et al. Robotic leg control with EMG decoding in an amputee with nerve transfers[J]. New England Journal of Medicine, 2013, 369(13): 1237-1242.

[14] HUANG H, ZHANG $\mathrm{F}$, HARGROVE L J, et al. Continuous locomotion-mode identification for prosthetic legs based on neuromuscular-mechanical fusion[J]. IEEE Transactions on Biomedical Engineering，2011，58(10): 2867-2875.

[15] SINGH R M, CHATTERJI S, KUMAR A. Trends and challenges in EMG based control scheme of exoskeleton robots-a review[J]. International Journal of Scientific and Engineering Research, 2012, 3(9): 933-940.

[16] FLEISCHER C, HOMMEL G. A human--exoskeleton interface utilizing electromyography[J]. IEEE Transactions on Robotics, 2008, 24(4): 872-882.

[17] KAWAMOTO H, TAAL S, NINISS H, et al. Voluntary motion support control of robot suit HAL triggered by bioelectrical signal for hemiplegia[C]// Engineering in Medicine and Biology Society (EMBC), 2010 Annual International Conference of the IEEE. IEEE, 2010: 462-466.

[18] KIGUCHI K, HAYASHI Y. An EMG-based control for an upper-limb power-assist exoskeleton robot[J]. IEEE Transactions on Systems, Man, and Cybernetics, Part B 
(Cybernetics), 2012, 42(4): 1064-1071.

[19] HOWARD M, BRAUN D J, VIJAYAKUMAR S. Transferring human impedance behavior to heterogeneous variable impedance actuators[J]. IEEE Transactions on Robotics, 2013, 29(4): 847-862.

[20] AJOUDANI A. Transferring human impedance regulation skills to robots[M]. Berlin: Springer, 2016.

[21] ISON M, VUJAKLIJA I, WHITSELL B, et al. High-density electromyography and motor skill learning for robust long-term control of a 7-DoF robot arm[J]. IEEE Transactions on Neural Systems and Rehabilitation Engineering, 2016, 24(4): 424-433.

[22] SENSINGER J W, LOCK B A, KUIKEN T A. Adaptive pattern recognition of myoelectric signals: Exploration of conceptual framework and practical algorithms[J]. IEEE Transactions on Neural Systems and Rehabilitation Engineering, 2009, 17(3): 270-278.

[23] YOUNG A J, HARGROVE L J, KUIKEN T A. The effects of electrode size and orientation on the sensitivity of myoelectric pattern recognition systems to electrode shift[J]. IEEE Transactions on Biomedical Engineering, 2011, 58(9): 2537-2544.

[24] MARIEB E N , HOEHN K. Human anatomy \& physiology[M]. Pearson Education, 2007.

[25] SHI J, ZHENG Y P, HUANG Q H, et al. Continuous monitoring of sonomyography, electromyography and torque generated by normal upper arm muscles during isometric contraction: Sonomyography assessment for arm muscles[J]. IEEE Transactions on Biomedical Engineering, 2008, 55(3): 1191-1198.

[26] ZHENG E, WANG L, WEI K, et al. A noncontact capacitive sensing system for recognizing locomotion modes of transtibial amputees[J]. IEEE Transactions on Biomedical Engineering, 2014, 61(12): 2911-2920.
[27] ZHENG E , WANG Q. Noncontact capacitive sensing-based locomotion transition recognition for amputees with robotic transtibial prostheses[J]. IEEE Transactions on Neural Systems and Rehabilitation Engineering, 2017, 25(2): 161-170.

[28] CAPPELLINI G, IVANENKO Y P, POPPELE R E, et al. Motor patterns in human walking and running[J]. Journal of Neurophysiology, 2006, 95(6): 3426-3437.

[29] WANG Q, YUAN K, ZHU J, et al. A robotic transtibial prosthesis with nonlinear damping behaviors for terrain adaptation[J]. IEEE Robotics and Automation Magazine, 2015(1): 375-379.

[30] GAO W, EMAMINEJAD S, NYEIN H Y Y, et al. Fully integrated wearable sensor arrays for multiplexed in situ perspiration analysis[J]. Nature, 2016, 529(7587): 509.

[31] KALTENBRUNNER M, SEKITANI T, REEDER J, et al. An ultra-lightweight design for imperceptible plastic electronics[J]. Nature, 2013， 499(7459): 458.

[32] KIM M, ALROWAIS H, PAVLIDIS S, et al. Size-scalable and high-density liquid-metal-based soft electronic passive components and circuits using soft lithography[J]. Advanced Functional Materials，2017，27(3): 1604466

[33] BURDET E, OSU R, FRANKLIN D W, et al. The central nervous system stabilizes unstable dynamics by learning optimal impedance[J]. Nature, 2001, 414(6862): 446.

作者简介: 王启宁(通信作者), 男, 1981 年出生, 博士, 研究员, 博士 研究生导师。主要研究方向为智能机器人和康复工程。

E-mail: qiningwang@pku.edu.cn

郑恩昊, 男, 1987 年出生, 博士, 副研究员。主要研究方向为神经接口、 人机交互。

E-mail: enhao.zheng@ia.ac.cn

许东方, 男, 1990 年出生, 博士研究生。主要研究方向为人机接口、仿 生材料。

E-mail: dongfangxu@pku.edu.cn

麦金耿, 男, 1982 年出生, 博士。主要研究方向为并行计算、神经接口。 E-mail: jingengmai@pku.edu.cn 